\title{
Speed of sound in the QGP and an SU(3) Yang-Mills theory
}

\author{
Z. V. Khaidukov, ${ }^{1,2}$ M. S. Lukashov, ${ }^{1,2}$ and Yu. A. Simonov ${ }^{1}$ \\ ${ }^{1}$ Institute for Theoretical and Experimental Physics, B. Cheremushkinskaya 25, Moscow 117259, Russia \\ ${ }^{2}$ Moscow Institute of Physics and Technology, Institutskiy per. 9, \\ Dolgoprudny, Moscow Region 141700, Russia
}

(Received 10 July 2018; published 31 October 2018)

\begin{abstract}
The speed of sound $C_{s}$ in the SU(3) and $(2+1)$ QCD is calculated within the field correlator method using the nonperturbative color magnetic confinement and Polyakov loop interaction in the deconfined region. The resulting $C_{S}$ displays a discontinuity at $T=T_{c}$ in the SU(3) case. It is shown numerically and analytically that $C_{s}^{2}$ never exceeds $\frac{1}{3}$ both for SU(3) and $(2+1)$ QCD for vanishing chemical potential. A good agreement is found of our numerical results with the corresponding lattice data.
\end{abstract}

DOI: 10.1103/PhysRevD.98.074031

\section{INTRODUCTION}

One of the most significant discoveries of recent times was experimental detection of quark-gluon plasma (QGP) [1-4]. It is the state of matter existing at extremely high temperatures in QCD. These conditions can be created in relativistic heavy-ion collisions [5-9].

In the framework of the research, it has been found that QGP, contrary to early theoretical predictions [10-15], is a liquid with an extremely low viscosity, but not a gas consisting of quarks and gluons. Therefore to describe processes in the heavy ions collision, (see e.g., [16] and references therein.) it is possible to use a hydrodynamic approach related to the physics of QGP $[17,18]$. One of the most important characteristics in hydrodynamics is the value of the speed of sound in the medium and its dependence on parameters (temperature, density, etc.). For example, it governs the evolution of the fireball produced in the heavy-ion collision, and one of the most important observables for describing of QGP formation-the elliptic flow [19-22].

The speed of sound is connected with the conformal symmetry breaking in hot QCD. In a scale-invariant system in the case of 3 spatial dimensions it should be $C_{s}^{2}=\frac{1}{3}$, because the trace of momentum-energy tensor must vanish $\epsilon-3 P=0$. It also can carry some information about the type of phase transition in the system.

The main source of information on the speed of sound in QCD is related with calculations on the lattice [23-27]. In the confined phase one can also use the hadron resonance gas model (HRG) [26,28,29]. The independent way to

Published by the American Physical Society under the terms of the Creative Commons Attribution 4.0 International license. Further distribution of this work must maintain attribution to the author(s) and the published article's title, journal citation, and DOI. Funded by SCOAP . obtain predictions for the speed of sound in QCD is connected with holographic description [30-37].

As it has become clear from numerous lattice and experimental studies of QCD at $T>0$, the main dynamics of both hadron and QGP phases is of the nonperturbative (np) origin and should be treated within the $n p$ methods.The fundamental approach to the np QCD is developed in the framework of the field correlator method (FCM) [38-43] generalized to nonzero temperatures [44-48]. In this paper we use the FCM to calculate the speed velocity in case of finite temperatures and taking into account the color magnetic confinement (CMC) as it was done in [49-53]. The strength of this method is the ability in a self-consistent manner to calculate the speed of sound both in the confinement phase and at temperatures above $T_{C}$ both at zero and nonzero chemical potential. The second case is very important because of the "sign problem" in lattice calculations in this domain. To circumvent this difficulty in the case of $N_{c}=3$ in QCD one finds Taylor coefficients in expansion around zero chemical potential to obtain the information about small densities [54,55], or uses imaginary chemical potential [56], or else considers the number of colors $N_{c}=2$, where this problem is absent [57-60]. In this work we will calculate the speed of sound in the case of pure $\mathrm{SU}(3)$ gluodynamics and also in the presence of quarks in $(2+1) \mathrm{QCD}$, for $\mu=0$.

The paper is organized as follows. In Sec. II we introduce the FCM in case of finite temperatures. In Secs. III and IV we use it to define the speed of sound as a function of the temperature in the case of pure Yang-Mills and of $(2+1)$ flavors QCD. Finally we summarize and discuss the obtained results in Sec. V.

\section{THE FIELD CORRELATOR METHOD}

The FCM is a useful instrument to treat the physics outside the area of perturbative theory. Analysis of physics 
of QGP in terms of FCM made in [44-48], has shown the important role of Polyakov loops for description of thermodynamic of QGP.Below we also take into account $\mathrm{CMC}$ effects, which are especially important at high $\mathrm{T}$. The main idea is as follows: The gluonic field $A_{\mu}$ splits into the background field $B_{\mu}$ and the (valence gluon) quantum field $a_{\mu}: A_{\mu}=B_{\mu}+a_{\mu}$, both satisfying the periodic boundary conditions. The partition function is:

$$
Z(B, T)=N \int D \phi \exp \left(-\int_{0}^{\beta} d t \int d^{3} x \mathcal{L}_{\text {tot }}\right),
$$

where $\phi$ denotes all set of fields $a_{\mu}, \psi, \psi^{+}$and ghost fields. In the lowest order in $g a_{\mu}$ one may obtain a result in the socalled single line approximation (SLA) $[47,48]$, where $q \bar{q}$ and $g g$ correlations are neglected

$$
\begin{aligned}
Z(B, T)= & N_{1}\left[\operatorname{det}\left(G^{-1}\right)\right]^{-\frac{1}{2}} \operatorname{det}\left(-D_{\lambda}^{2}(B)\right) \\
& \times\left[\operatorname{det}\left(m_{q}^{2}-\hat{D}^{2}(B)\right)\right]^{1 / 2}
\end{aligned}
$$

where $N_{1}$-normalization factor, $D_{\lambda}(B)=\partial_{\lambda}-i g B_{\lambda}, G^{-1}=$ $D_{\lambda}^{2} \delta_{\mu \nu}+2 i g F_{\mu \nu}$. The thermodynamic potential $\mathrm{F}(\mathrm{T})$ is connected to $\mathrm{Z}(\mathrm{B}, \mathrm{T})$ in standard way

$$
F(T)=-T \ln (Z(B))_{B}
$$

where index B means averaging over all background fields. In SLA the contributions of gluons and quarks in $F(T)$ are separated:

$$
F(T)_{S L A}=F_{q}(T)+F_{g l}(T) .
$$

In FCM the breaking of Lorentz invariance at finite temperatures becomes apparent through existence of two types of string tension:

$$
\sigma^{E, H}=\frac{1}{2} \int D^{E, H} d^{2} z
$$

where $D^{E, H}$ is obtained from

$$
\begin{aligned}
& \frac{g^{2}}{N_{c}}\langle\left.\left.\operatorname{Tr} E_{i}(x) \Phi E_{j}(y) \Phi^{+}\right\rangle\right\rangle \\
&= \delta_{i j}\left(D^{E}(u)+D_{1}^{E}(u)+u_{4}^{2} \frac{\partial D_{1}^{E}(u)}{\partial u^{2}}\right) \\
&+u_{i} u_{j} \frac{\partial^{2} D_{1}^{E}(u)}{\partial u^{2}} \\
& \frac{g^{2}}{N_{c}}\left\langle\left\langle\operatorname{Tr} H_{i}(x) \Phi H_{j}(y) \Phi^{+}\right\rangle\right. \\
&=\delta_{i j}\left(D^{H}(u)+D_{1}^{H}(u)+\mathbf{u}^{2} \frac{\partial D_{1}^{H}(u)}{\partial \mathbf{u}^{2}}\right) \\
& \quad-u_{i} u_{j} \frac{\partial^{2} D_{1}^{H}(u)}{\partial u^{2}},
\end{aligned}
$$

where $u=x-y$ and $\Phi(x, y)=P \exp \left(\int_{y}^{x} A_{\mu} d z^{\mu}\right)$. At zero temperature both string tensions $\left(\sigma^{E}=\sigma^{H}=\sigma\right)$ coincide and $\sigma$ forms the basic np scale, which defines all hadron masses and the QCD scale in general. The values of $\sigma^{E, H}$ can be also obtained from calculations on the lattice (see e.g., [50]).

The correlators $D^{E}$ and $D_{1}^{E}$ produce both the scalar confining interaction $V_{D}(r)$ and the vector-like interaction $V_{1}(r)$ :

$$
\begin{gathered}
V_{D}(r)=2 c_{\alpha} \int_{0}^{r}(r-\lambda) d \lambda \int_{0}^{\infty} d \nu D^{E}(\lambda, \nu) \\
=V_{D}^{\operatorname{lin}}(r)+V_{D}^{\mathrm{sat}}(r) \\
V_{1}(r)=c_{\alpha} \int_{0}^{r} \lambda d \lambda \int_{0}^{\infty} d \nu D_{1}^{E}(\lambda, \nu), c_{\text {fund }}=1, c_{\text {adj }}=\frac{9}{4}
\end{gathered}
$$

From $V_{D}(r)$ we extract the purely linear form $V_{D}^{\operatorname{lin}}(r)$, and for $V_{1}(r)$ we separate out the one gluon exchange, $V^{\text {oge }}$, $V_{1}(r)=V_{1}^{\text {sat }}+V^{\text {oge }}$, while the rest parts, $V_{d}^{\text {sat }}$ and $V_{1}^{\text {sat }}$ are saturating at large $\mathrm{r}$, thus for the total potential below $T_{c}$ one obtain:

$V\left(r, T<T_{c}\right)=V_{D}^{\mathrm{lin}}(r)+V_{1}^{\mathrm{sat}}(r)+V^{\mathrm{oge}}(r)+V_{D}^{\mathrm{sat}}(r)$

It is worth emphasizing that at low temperatures $V_{D}^{\text {sat }}(r)$ and $V_{1}^{\text {sat }}$ compensate each other (for details, see Appendix of [50]). But at temperatures above $T_{c}, V_{D}(r)$ vanishes. As for $V_{1}^{\text {sat }}(r, T)$, this quantity defines Polyakov loops $\left(L_{i}\right)$, i.e:

$L_{i}=\exp \left(-c_{i} \frac{V_{1}(\infty, T)}{2 T}\right), c_{\text {fund }}=1, c_{\text {adj }}=\frac{9}{4}, i=\operatorname{adj}, f$

The contribution of Polyakov loops alone gives a reasonable agreement with the lattice results [47]. However for an accurate description of data one needs the CMC ingredient which we introduce below following [48]. The relationship between pressure, volume, and free energy is given by:

$$
P_{g l} V_{3}=-F_{0}(B)
$$

For the gluon contribution we obtain:

$$
\begin{aligned}
P_{g l} & =2\left(N_{c}^{2}-1\right) \int_{0}^{\infty} \frac{d s}{s} \sum_{n \neq 0} G^{n}(s), G^{n}(s) \\
& =\int(D z)_{\mathrm{on}}^{\omega} e^{-K} \hat{t r}_{a}\left\langle W_{\Sigma}^{a}\left(C_{n}\right)\right\rangle
\end{aligned}
$$




$$
\begin{aligned}
K= & \frac{1}{4} \int_{0}^{s} d \tau\left(\frac{d z^{\mu}}{d \tau}\right)^{2}, \\
(D z)_{x y}^{\omega}= & \lim _{N \rightarrow \infty} \prod_{m=1}^{n} \frac{d^{4} \zeta(m)}{(4 \pi \epsilon)^{2}} \sum_{n=0, \pm, \ldots} \frac{d^{4} p}{(2 \pi)^{4}} \\
& \times \exp \left[i p_{\mu}\left(\sum_{m=1}^{N} \zeta(m)-(x-y)_{\mu}-n \beta \delta_{\mu 4}\right)\right]
\end{aligned}
$$

where we use the Fock-Feynman-Schwinger (FFS) formalism with Schwinger proper time s [48]. $W_{\Sigma}^{a}\left(C_{n}\right)$ is the adjoint Wilson loop defined for the gluon path $C_{n}$, which has both temporal (i4) and spacial projections (ij), and $\hat{t} r_{a}$ is the normalized adjoint trace.

$\mathrm{CE}$ and CM fields strengths in $T>T_{c}$ region correlate very weakly due go the gauge-invariant field correlator in adjoint representation $\left\langle E_{i}(x) B_{k}(y) \Phi(x, y)\right\rangle \approx 0$ (see $[47,48])$ and therefore both $\mathrm{CE}$ and $\mathrm{CM}$ projections of the $\operatorname{tr}_{a} W_{\Sigma}^{a}\left(C_{n}\right)$ can be factorized as shown in [50]

$$
\left\langle W_{\Sigma}^{a}\left(C_{n}\right)\right\rangle=L_{\mathrm{adj}}^{(n)}(T)\left\langle W_{3}\right\rangle,
$$

for $L_{i}^{(n)} \approx L_{i}^{n}$ for $T \leq 1 \mathrm{GeV}$, One can integrate out the $z_{4}$ part of the path integral $(D z)_{\mathrm{on}}^{\omega}=\left(D z_{4}\right)_{\mathrm{on}}^{\omega} D^{3} z$, and write the result as

$$
\begin{aligned}
G^{(n)}(s) & =G_{4}^{(n)}(s) G_{3}(s), \\
G_{4}^{n}(s) & =\int\left(D z_{4}\right)_{\mathrm{on}}^{\omega} e^{-K} L_{\text {adj }}^{(n)}=\frac{1}{2 \sqrt{4 \pi s}} e^{-\frac{n^{2}}{4 T^{2} s}} L_{\text {adj }}^{(n)}
\end{aligned}
$$

For the Polyakov loops, one can obtain [44]:

$$
\begin{aligned}
L_{\text {adj }}^{n} & =\exp \left(-\frac{9}{4} J_{n}^{E}\right), \\
J_{n}^{E} & =\frac{n \beta}{2} \int_{0}^{n \beta} d \nu\left(1-\frac{\nu}{n \beta}\right) \int_{0}^{\infty} \zeta d \zeta D_{1}^{E}\left(\sqrt{\zeta^{2}+\nu^{2}}\right)
\end{aligned}
$$

and finally

$$
\begin{gathered}
P_{g l}=\frac{N_{c}^{2}-1}{\sqrt{4 \pi}} \int_{0}^{\infty} \frac{d s}{s^{3 / 2}} G_{3}(s) \sum_{n= \pm 1, \pm 2, \ldots} e^{-\frac{n^{2}}{4 T^{2} s}} L_{\mathrm{adj}}^{n} \\
G_{3}(s)=\int\left(D^{3} z\right)_{x x} e^{-K_{3 d}}\left\langle\hat{t} r_{a} W_{3}^{a}\right\rangle
\end{gathered}
$$

In a similar way one can consider the quark contribution:

$$
\begin{aligned}
P_{q} & =2 N_{c} \int_{0}^{\infty} \frac{d s}{s} e^{-m_{q}^{2} s} \sum_{n=1}^{\infty}(-1)^{n+1}\left[S_{n}(s)+S_{-n}(s)\right], S_{n}(s) \\
& =\frac{1}{N_{c}} \int(D z)_{o n}^{\omega} e^{-K} \hat{t r}\left\langle W_{\sigma}\left(C_{n}\right)\right\rangle
\end{aligned}
$$

and the pressure acquires the form:

$$
\begin{gathered}
P_{q}=\frac{4 N_{c}}{\sqrt{4 \pi}} \int_{0}^{\infty} \frac{d s}{s^{3 / 2}} e^{-m_{q}^{2} s} S_{3}(s) \sum_{n=1,2, \ldots}(-)^{n+1} e^{-\frac{n^{2}}{4 T^{2} s}} L_{f}^{n} \\
S_{3}(s)=\int\left(D^{3} z\right)_{x x} e^{-K_{3 d}}\left\langle\hat{t} r_{f} W_{3}^{f}\right\rangle
\end{gathered}
$$

Equations (18), (16) provide a general expression for the free energy $-F=P_{g}+P_{q}$ and we can find all thermodynamic quantities and their dependence on parameters of the media.

It is necessary to make two important remarks. (1) From the factor $\exp \left(-\frac{n^{2}}{4 T^{2} s}\right)$ and also from the contribution of CMC follows the suppression of high order terms in (16), (19). (2) The contribution of CMC in single line approximation dictates the form of the propagator $G_{3}(s)$ and defines the screening masses $M_{D}$ in it. The latter, as shown in $[49,50]$ is growing with $\mathrm{T}$ and defines all dynamics at large $\mathrm{T}$.

\section{THE SPEED OF SOUND IN SU(3) YANG-MILLS THEORY}

We start with the speed of sound in the case of SU(3) gluodynamics. The Lagrangian is

$$
\begin{gathered}
\mathcal{L}=-\frac{1}{4} G_{\mu \nu}^{a} G_{\mu \nu}^{a} \\
G_{\mu \nu}^{a}=\partial_{\mu} A_{\nu}^{a}-\partial_{\nu} A_{\mu}^{a}+g f^{a b c} A_{\mu}^{b} A_{\nu}^{c}
\end{gathered}
$$

where $G_{\mu \nu}^{a}$ is non-Abelian field strength, $a=1, \ldots$, $N^{2}-1, \mu, \nu=1 . .4$ In this model there is a confinementdeconfinement phase transition, of the weak first order, from the phase of the glueball gas to the gluon plasma as known from lattice studies (see e.g., [27]), and from the FCM analysis [48]). In addition to the gauge symmetry of the Lagrangian (21), there is also a scale symmetry of the Lagrangian on the classical level,

$$
x \rightarrow \lambda^{-1} x, A_{\mu}^{a}(x) \rightarrow \lambda A_{\mu}^{a}(x)
$$

As a consequence, the trace of the energy-momentum tensor must vanish $\left\langle T_{\mu \mu}\right\rangle=0$. One might expect that in the thermodynamic description the equality $E=3 P$ (where $E$ is the system energy and $P$ is the system pressure) holds. However, it is well known that inclusion of quantum effects for non-Abelian fields leads to the appearance of a mass scale. From the lattice calculations we know that the scaling symmetry in SU(3) Yang-Mills is significantly violated, especially in the confinement-deconfinement transition area [61]. As mentioned in the introduction, the speed of sound is an excellent indicator for this violation, therefore we expect to obtain in our calculations that the speed of sound is different from $1 / 3$ in the vicinity of $T_{c}$. 
The second important fact is related to the type of transition. In [62], it was suggested that in case of pure Yang-Mills the type of confinement-deconfinement transition depends on the number of colors. In case of $N_{c}=2$ there must be a second-order phase transition [62], while in the case of $N_{c}=3$ it is of the first order [27,63]. From the expression

$$
C_{s}^{2}=\frac{s}{\frac{\partial \epsilon}{\partial T}}=\frac{\frac{\partial P}{\partial T}}{\frac{\partial \epsilon}{\partial T}}
$$

one can see that at the point $T=T_{c}$ a possible discontinuity in the speed of sound that is confirmed by calculations on the lattice [61].

The introduction of FCM for QCD was described in the previous section and will be used below for numerical calculations. In addition one can provide a qualitative analysis of resulting equations. One can use Eq. (16) and obtain the energy density:

$$
\epsilon+P=T \frac{\partial P}{\partial T} .
$$

Writing $P=T^{4} f(T)$ one has:

$$
\begin{aligned}
C_{s}^{2}=\frac{\frac{\partial P}{\partial T}}{T \frac{\partial^{2} P}{\partial T^{2}}} & =\frac{1}{3} \frac{f+\frac{1}{4} T f^{\prime}(T)}{f+\frac{2}{3} T f^{\prime}(T)+\frac{1}{12} T^{2} f^{\prime \prime}(T)} \\
& \approx \frac{1}{3}\left(1-\frac{5}{12} \frac{T f^{\prime}(T)}{f(T)}+O\left(T^{2} f^{\prime \prime}\right)\right) .
\end{aligned}
$$

To understand the behavior of $f(T)$ we use [45] for $G_{3}(s)$

$$
P_{g l}=\frac{2\left(N_{c}^{2}-1\right)}{16 \pi^{2}} \sum_{n=1}^{\infty} L_{\text {adj }}^{n} \int_{0}^{\infty} \frac{d s}{s^{3}} e^{-\frac{n^{2}}{4 T^{2}} s} \phi\left(M_{0}^{2} s\right)
$$

where $\phi=\left(\frac{M_{0}^{2} s}{s h\left(M_{0}^{2} s\right)}\right)^{\gamma}, \gamma=1$ for oscillator form of color magnetic confinement, and $\gamma=1 / 2$ for the linear confinement. The screening mass $M_{0}$ is expressed via spacial string tension, $M_{0}^{2}=a \sigma_{s}, a=8 \gamma$, and $\sigma_{s}$ was obtained from the lattice data [61]:

$$
\sigma_{s}(T)=c_{\sigma}^{2} g^{4}(T) T^{2}, \quad c_{\sigma}=0.566 \pm 0.013 .
$$

Changing variables in the integral (27) one obtains:

$$
I_{n}\left(\kappa^{2}\right)=\int_{0}^{\infty} \frac{d u}{u^{3}} e^{-\frac{n^{2}}{4 u}} \phi\left(u \kappa^{2}\right), \quad \kappa=\frac{M_{0}^{2}}{T^{2}}
$$

$f(T)$ can be written as:

$$
f(T)=\frac{2\left(N_{c}^{2}-1\right)}{16 \pi^{2}} \sum_{n=1}^{\infty} L_{\mathrm{adj}}^{n} I_{n}\left(\kappa^{2}\right) .
$$

The derivative $f^{\prime}(T)$ consists of two terms $\frac{\partial L_{\text {adj }}^{n}}{\partial T}$ and $\frac{\partial I_{n}\left(\kappa^{2}\right)}{\partial T}=I_{n}^{\prime}\left(\kappa^{2}\right) \frac{\partial \kappa^{2}}{\partial T}, \frac{\partial \kappa^{2}}{\partial T}=8 \gamma c_{\sigma}^{2} \frac{\partial g^{4}}{\partial T}$, where:

$$
I_{n}^{\prime}\left(\kappa^{2}\right)=\int_{0}^{\infty} \frac{d u}{u^{3}} e^{-\frac{n^{2}}{4 u}} \frac{\partial \phi}{\partial \kappa^{2}} \frac{\partial \kappa^{2}}{\partial T}
$$

and one obtains that both $\frac{\partial \phi}{\partial \kappa^{2}}$ and $\frac{\partial \kappa^{2}}{\partial T}$ are negative, so that $\frac{\partial I}{\partial T}>0$. The same conclusion follows for $\frac{\partial L_{i}}{\partial T}$,

$$
\frac{\partial L_{\text {adj }}^{n}}{\partial T}=\frac{\partial}{\partial T}\left(e^{\frac{-9 n V_{1}(\infty, T)}{8 T}}\right)>0
$$

where $V_{1}(\infty, T)$ decreases with $\mathrm{T}$ for $T>T_{c}$, while $L_{\text {adj }}^{n}$ grows with T. Hence one can deduce that: $f^{\prime}(T)>0$, for $T>T_{c}$ and consequently:

$$
C_{s}^{2}-\frac{1}{3} \approx-\frac{5}{36} \frac{f^{\prime}(T)}{f(T)}<0
$$

Note, that the largest contribution to $f^{\prime}(T)$ comes from $\frac{\partial L_{\text {adj }}^{n}}{\partial T}$.

In the confinement area, $T<T_{c}$ we can also make some predictions. For the pressure of glueball resonans gas one can write:

$f_{g b}=\sum_{i} \frac{g_{i}}{2 \pi^{2}} \sum_{n=1}^{\infty} \frac{m_{i}^{2}(T)}{n^{2} T^{2}} K_{2}\left(\frac{n m_{i}}{T}\right) \equiv \sum_{i} \frac{g_{i}}{2 \pi^{2}} \sum_{n=1}^{\infty} \frac{1}{n^{4}} k_{n}^{(i)}$

$$
k_{n}^{(i)}(T) \equiv \frac{n^{2} m_{i}^{2}(T)}{T^{2}} K_{2}\left(\frac{n m_{i}}{T}\right) .
$$

In (34), (35) one can take into account, that confinement and string tension $\sigma(T)$ are $T$ dependent [50], so that

$$
m_{i}(T)=a(T) m_{i}(0), \quad a(T)=\sqrt{\frac{\sigma_{E}(T)}{\sigma_{E}(0)}} .
$$

Note, that $k_{n}^{(i)}(T \rightarrow \infty) \rightarrow \frac{1}{2}$, while for small $T, T \ll m_{i}(T)$ one has

$k_{n}^{(i)}(T \rightarrow 0)=\sqrt{\frac{\pi}{2}}\left(\frac{n m_{i}(0)}{T}\right)^{3 / 2} \exp \left(-\frac{n m_{i}(0)}{T}\right)$

As a consequence for $T \ll m_{i}(0)$ the main contribution comes from the term with the lowest mass, and thus from (35):

$$
C_{s}^{2}=\frac{T}{m_{0}}, T \rightarrow 0 .
$$

As one can see in Fig. 1 there is a good agreement between the predictions of the theory Eqs. (16), (24) 


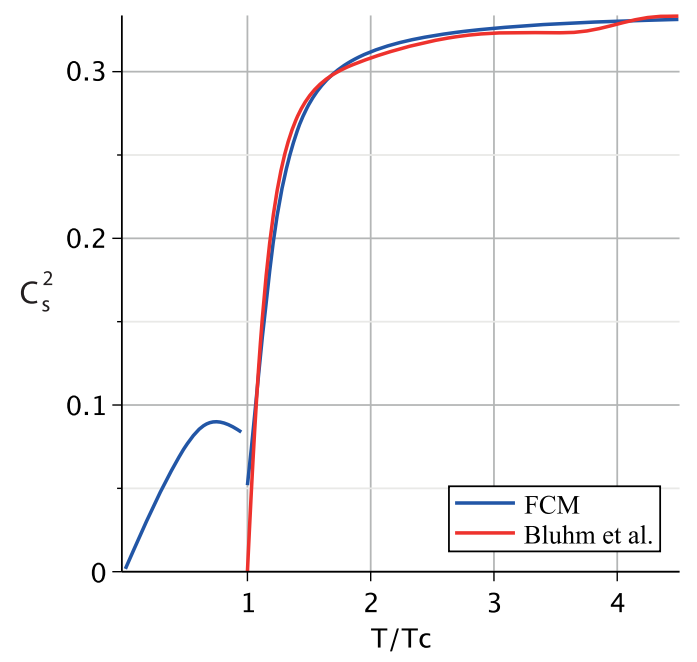

FIG. 1. The speed of sound in FCM from (24) for SU(3) in comparison with the fit from [64].

and the fit from [64]. We preferred to show this fit instead of lattice data because there is a good agreement between this fit and the lattice data from $[61,65]$. We also obtain discontinuity at $T=T_{c}$, as it should be in case of the first order phase transition.

\section{THE SPEED OF SOUND IN QCD WITH (2 + 1) FLAVORS}

Here we shall consider the speed of sound in case of QCD with $n_{f}=2+1$. We take $m_{u}, m_{d}$ equal to zero and $m_{s}=0.1 \mathrm{GeV}$.

As in the case of the Yang-Mills theory, some qualitative predictions about the value of the speed of sound can be made. First of all, because the confinement-deconfinement phase transition in this case is a crossover [56], the speed of sound can have a finite value and a local minimum in the crossover region. The second prediction is connected with the domain of extremely large $\mathrm{T}$, where $C_{s}$ should be close to $1 / \sqrt{3}$, because in this region the scale anomaly is small relative to the typical energy scale [26].

Similarly to the way it was done for $P_{g}$ in the last chapter, we can consider $P_{q}$, Eq. (19) for the ensemble of quarks. We use the pressure from [52].

$$
\begin{aligned}
P_{q}= & \sum_{f} P_{q}^{(f)}, \\
P_{q}^{(f)}= & \frac{4 N_{c}}{(4 \pi)^{2}} \int \frac{d s}{s^{3}} e^{-m_{f}^{2} s} \\
& \times \sum_{n=1,2}(-)^{n+1} e^{-\frac{n^{2}}{4 T^{2} s}} \cosh \left(\frac{\mu n}{T}\right) L_{f}^{(n)} \varphi_{f}(s) \\
\varphi_{f}(s)= & \left(\frac{M_{f}^{2} s}{\sinh \left(M_{f}^{2} s\right)}\right)^{\gamma}, \quad M_{f}^{2}=\frac{4}{9} M_{0}^{2}=\frac{4}{9} a \sigma_{s}
\end{aligned}
$$

Similarly to (27), one obtains for $f_{f}(T)$ at $\mu=0$

$$
f_{f}(T)=\frac{4 N_{c}}{(4 \pi)^{2}} \sum_{n=1}^{\infty} L_{f}^{(n)} I_{n}^{(f)}\left(\kappa^{2}\right) e^{-\frac{m_{f}^{2}}{T^{2}} u}
$$

with

$$
I_{n}^{(f)}=\int_{0}^{\infty} \frac{d u}{u^{3}} e^{-\frac{n^{2}}{4 u}} \varphi_{f}\left(u \kappa_{f}^{2}\right), \quad \kappa_{f}^{2}=\frac{M_{f}^{2}}{T^{2}}
$$

Writing now $C_{s}^{2}-\frac{1}{3}=-\Delta_{q g}$, with $\Delta_{q g}(T)=\frac{5}{36} T\left(\frac{f_{g}^{\prime}(T)}{f}+\right.$ $\left.\frac{f_{f}^{\prime}(T)}{f}\right)$ and neglecting the relatively small $\mathrm{T}$ derivatives of $\kappa_{f, g}^{2}=\frac{M_{f, g}^{2}}{T^{2}}$, one obtains for small $\frac{m_{f}}{T}$

$$
\begin{aligned}
\Delta_{q g} \cong & \frac{5}{36}\left\{\sum_{f} \sum_{n} \frac{4 N_{c}}{(4 \pi)^{2}} e^{-\frac{m_{f}^{2}}{T^{2}} u} I_{n}^{(f)}\left(T \frac{\partial L_{f}^{(n)}}{\partial T}\right)\right. \\
& \left.+\sum_{n} \frac{2\left(N_{c}^{2}-1\right)}{(4 \pi)^{2}} I_{n}\left(\kappa^{2}\right)\left(T \frac{\partial L_{f}^{(n)}}{\partial T}\right)\right\} .
\end{aligned}
$$

One can see that always $\Delta_{q g}>0$ and at large $T$, $T>250 \mathrm{MeV}, \Delta_{q g}$ is small and tends to zero. In a similar way as for the glueball gas, one can treat the hadron resonance gas of mesons and baryons. Neglecting interaction between hadrons, one arrives at the small $\mathrm{T}$ limit (38) for the sound velocity of the HRG with the pion mass for $m_{0}$

We compare our results with [26] and [66] in Fig. 3.

\section{NUMERICAL RESULTS AND DISCUSSIONS}

To understand the behavior of $C_{s}^{2}$ as a function of $\mathrm{T}$ and compare it with our qualitative predictions (33) and (43), as well as with lattice calculations, we have computed $P_{g l}(T)$

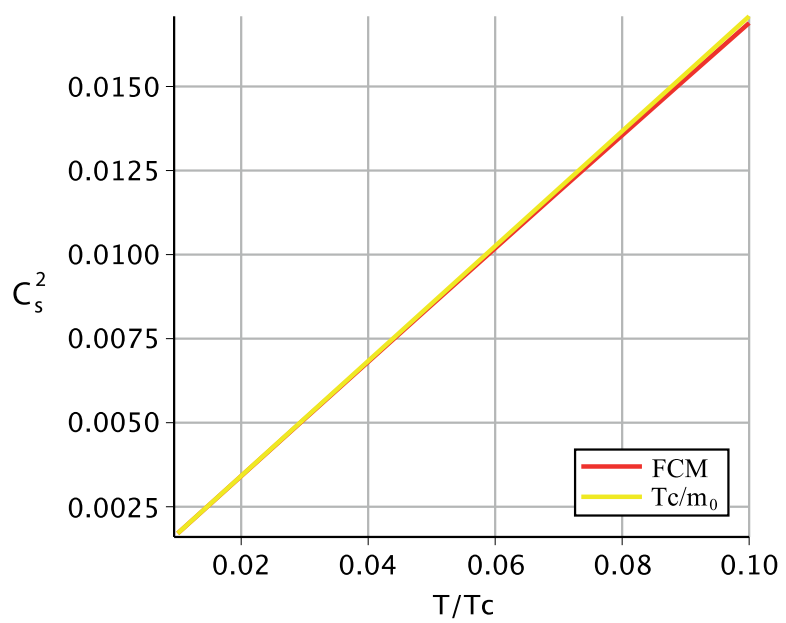

FIG. 2. The speed of sound $C_{s}^{2}$ in FCM from (24) for SU(3) in the limit $T \rightarrow 0$ in comparison with (38). 


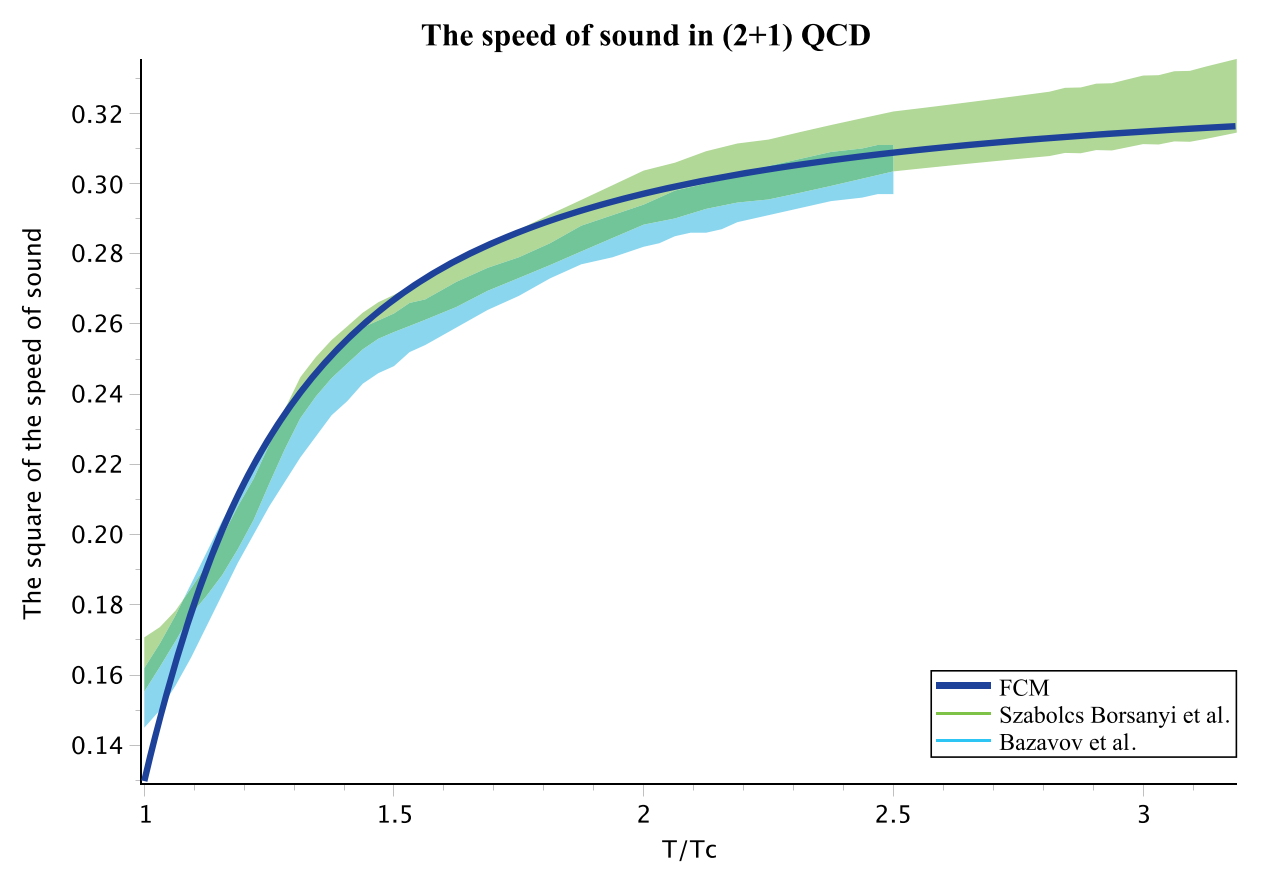

FIG. 3. The speed of sound in QGP from FCM, Eqs. (24), (16), and (19), in comparison with lattice data of Borsanyi et al. [66] and Bazavov et al. [26].

Eq. (27) with $\gamma=1 / 2$ and obtain $C_{s}^{2}$, using the definition (24) The results for $C_{s}^{2}$ are plotted in Fig. 1 in comparison with the lattice data from [64]. One can see a good agreement with lattice curve for $C_{s}^{2}$ and a discontinuity at $T=T_{c}$, as should be for the first order SU(3) transition. Both lattice and our results for $C_{s}^{2}$ are in the region $C_{s}^{2} \leq 1 / 3$, which supports our qualitative conclusion in (33). It is interesting to study the behavior of $C_{s}^{2}$ at $T \rightarrow 0$, which was done in Fig. 2, where the limiting relation (38) is compared with our numerical data, showing a perfect agreement. For the $2+1$ QCD our numerical data are presented in Fig. 3 in comparison with the lattice data of $[26,55]$. One can see again a good agreement of all results. Comparing with our analytic predictions in (43), $C_{s}^{2}-\frac{1}{3}=-\Delta_{q g}$, one finds that indeed $\Delta_{q g}$ is positive in the whole region $T>T_{c}$, and it is small for $T>250 \mathrm{MeV}$.
Thus for the QGP one reveals the behavior, of the squared sound velocity, which never exceeds $1 / 3$ and can be called normal. However, already at nonzero $\mu$ one might meet with a new phenomenon, since $\mu$ enters $P_{q}$, as in (39) via $\cosh \frac{\mu n}{T}$, and this provides a negative sign of $\Delta_{q g}$ for large enough $\mu / T$. One can expect also a strong deviation of $C_{s}^{2}$ in the presence of external magnetic fields. Both this effects require additional studies and will be subject of further publications.

\section{ACKNOWLEDGMENTS}

The authors are grateful for useful discussions to M. A. Andreichikov and B. O. Kerbikov, M. A. Zubkov. This work was done in the frame of the scientific project, supported by the Russian Science Foundation Grant No. 16-12-10414.
[1] J. Adams et al. (STAR Collaboration), Nucl. Phys. A757, 102 (2005).

[2] K. Adcox et al. (PHENIX Collaboration), Nucl. Phys. A757, 184 (2005).

[3] I. Arsene et al., Nucl. Phys. A757, 1 (2005).

[4] M. Gyulassy and L. McLerran, Nucl. Phys. A750, 30 (2005).

[5] B. B. Back et al. (PHOBOS Collaboration), Nucl. Phys. A757, 28 (2005).
[6] E. V Shuryak, Rev. Mod. Phys. 89, 035001 (2017).

[7] P. Braun-Munzinger, V. Koch, T. Schafer, and J. Stachel, Phys. Rep. 621, 76 (2016).

[8] W. Busza, K. Rajagopal, and W. van der Schee, Massachusetts Institute of Technology, Center for Theoretical Physics, Report No. MIT-CTP/4892 [arXiv:1802.04801].

[9] R. Pasechnik and M. Sumbera, Universe 3, 7 (2017).

[10] R. D. Pisarski and F. Wilczek, Phys. Rev. D 29, 338 (1984). 
[11] G. F. Chapline, M. H. Johnson, E. Teller, and M. S. Weiss, Phys. Rev. D 8, 4302 (1973).

[12] J. C. Collins and M. J. Perry, Phys. Rev. Lett. 34, 1353 (1975).

[13] T. D. Lee, Phys. Rev. D 19, 1802 (1979).

[14] N. Cabibbo and G. Parisi, Phys. Lett. 59B, 67 (1975).

[15] E. V. Shuryak, Zh. Eksp. Teor. Fiz. 74, 408 (1978) [Sov. Phys. JETP 47, 212 (1978)].

[16] R. Snellings, New J. Phys. 13, 055008 (2011).

[17] D. A. Teaney, arXiv:0905.2433.

[18] T. Hirano and M. Gyulassy, Nucl. Phys. A769, 71 (2006).

[19] J. Ollitrault, Phys. Rev. D 46, 229 (1992).

[20] H. Sorge, Phys. Rev. Lett. 82, 2048 (1999).

[21] P. Kolb, J. Sollfrank, and U. Heinz, Phys. Lett. B 459, 667 (1999).

[22] D. Teaney, J. Lauret, and E. Shuryak, Phys. Rev. Lett. 86, 4783 (2001).

[23] R. V. Gavai, S. Gupta, and S. Mukherjee, Phys. Rev. D 71, 074013 (2005).

[24] F. Karsch, J. Phys. Conf. Ser. 46, 122 (2006).

[25] O. Philipsen, Prog. Part. Nucl. Phys. 70, 55 (2013).

[26] A. Bazavov, T. Bhattacharya, C. DeTar, H.-T. Ding, S. Gottlieb, R. Gupta, P. Hegde, U. M. Heller, F. Karsch, E. Laermann, L. Levkova, S. Mukherjee, P. Petreczky, C. Schmidt, C. Schroeder, R. A. Soltz, W. Soeldner, R. Sugar, M. Wagner, and P. Vranas (HotQCD Collaboration), Phys. Rev. D 90, 094503 (2014).

[27] S. Borsanyi, G. Endrodi, Z. Fodor, A. Jakovác, S. D. Katz, S. Krieg, C. Ratti, and K. K. Szabó, J. High Energy Phys. 11 (2010) 077.

[28] R. Venugopalan and M. Prakash, Nucl. Phys. A546, 718 (1992).

[29] M. Bluhm, P. Alba, W. Alberico, A. Beraudo, and C. Ratti, Nucl. Phys. A929, 157 (2014).

[30] R. Rougemont, R. Critelli, and J. Noronha, Phys. Rev. D 93, 045013 (2016).

[31] R. Rougemont, A. Ficnar, S. Finazzo, and J. Noronha, J. High Energy Phys. 04 (2016) 102.

[32] P. M. Hohler and M. A. Stephanov, Phys. Rev. D 80, 066002 (2009).

[33] S. S. Gubser and A. Nellore, Phys. Rev. D 78, 086007 (2008).

[34] U. Gursoy, E. Kiritsis, L. Mazzanti, and F. Nitti, Nucl. Phys. B820, 148 (2009).

[35] S. S. Gubser, A. Nellore, S. S. Pufu, and F. D. Rocha, Phys. Rev. Lett. 101, 131601 (2008).

[36] S. S. Gubser and A. Nellore, Phys. Rev. D 78, 086007 (2008).

[37] O. Andreev, Phys. Rev. D 76, 087702 (2007).

[38] H. G. Dosch, Phys. Lett. B 190, 177 (1987).

[39] H. G. Dosch and Yu. A. Simonov, Phys. Lett. B 205, 339 (1988).
[40] Yu. A. Simonov, Nucl. Phys. B307, 512 (1988).

[41] A. Di Giacomo, H. G. Dosch, V. I. Shevchenko, and Yu. A. Simonov, Phys. Rep. 372, 319 (2002).

[42] Yu. A. Simonov, Phys. Usp. 39, 313 (1996).

[43] D. S. Kuzmenko, V. I. Shevchenko, and Yu. A. Simonov, Phys. Usp. 174, 3 (2004).

[44] Yu. A. Simonov, Phys. Lett. B 619, 293 (2005).

[45] N. O. Agasian and Yu. A. Simonov, Phys. Lett. B 639, 82 (2006).

[46] Yu. A. Simonov and M. A. Trusov, Phys. Lett. B 650, 36 (2007).

[47] E. V. Komarov and Yu. A. Simonov, Ann. Phys. (Amsterdam) 323, 1230 (2008).

[48] Yu. A. Simonov, Ann. Phys. (Amsterdam) 323, 783 (2008).

[49] M. S. Lukashov and Yu. A. Simonov, JETP Lett. 105, 691 (2017).

[50] N. O. Agasian, M. S. Lukashov, and Yu. A. Simonov, Eur. Phys. J. A 53, 138 (2017).

[51] N. O. Agasian, M. S. Lukashov, and Yu. A. Simonov, Mod. Phys. Lett. A 31, 1650222 (2016).

[52] M. A. Andreichikov, M. S. Lukashov, and Yu. A. Simonov, Int. J. Mod. Phys. A 33, 1850043 (2018).

[53] Yu. A. Simonov, Phys. Rev. D 96, 096002 (2017).

[54] A. Bazavov, H.-T. Ding, P. Hegde et al., Phys. Rev. D 95, 054504 (2017).

[55] S. Borsanyi, G. Endrodi, Z. Fodor, S. D. Katz, S. Krieg, C. Ratti, and K. K. Szabo, J. High Energy Phys. 08 (2012) 053.

[56] J. Gunther, R. Bellwied, S. Borsanyi, Z. Fodor, S. D. Katz, A. Pasztor, and C. Ratti, Nucl. Phys. A967, 720 (2017).

[57] V. G. Bornyakov, V. V. Braguta, E.-M. Ilgenfritz, A. Kotov, A. Molochkov, and A. Nikolaev, J. High Energy Phys. 03 (2018) 161.

[58] V. Braguta, E. Ilgenfritz, A. Kotov, A. Molochkov, and A. Nikolaev, Phys. Rev. D 94, 114510 (2016).

[59] V. V. Braguta, E.-M. Ilgenfritz, A. Yu. Kotov, B. Petersson, and S. A. Skinderev, Phys. Rev. D 93, 034509 (2016).

[60] V. V. Braguta, V. A. Goy, E.-M. Ilgenfritz, A. Yu. Kotov, A. V. Molochkov, M. Müller-Preussker, and B. Petersson, J. High Energy Phys. 06 (2015) 094.

[61] G. Boyd, J. Engels, F. Karsch, E. Laermann, C. Legeland, M. Luetgemeier, and B. Petersson, Nucl. Phys. B469, 419 (1996).

[62] B. Svetitsky and G. Yaffe, Nucl. Phys. B210, 423 (1982).

[63] T. Celik, J. Engels, and H. Satz, Phys. Lett. B 125, 411 (1983).

[64] M. Bluhm, B. Kampfer, and K. Redlich, Phys. Rev. C 84, 025201 (2011).

[65] M. Okamoto, A. Ali Khan, S. Aoki et al., Phys. Rev. D 60, 094510 (1999).

[66] S. Borsanyi, Z. Fodor, and C. Hoelbling, Phys. Lett. B 730, 99 (2014). 